\title{
Optical properties of InGaNAs/GaAs quantum well structures with GaNAs strain relief buffer layers
}

\author{
Cheng-Yuan Chen ${ }^{\mathrm{a}}$, Jia-Ren Lee ${ }^{\mathrm{b}}$, Chien-Rong Lu ${ }^{\mathrm{a}, *}, \mathrm{Hsiang}-\mathrm{Lin} \mathrm{Liu}^{\mathrm{a}}$, \\ Li-Wen Sun ${ }^{\mathrm{c}}$, Hao-Hsiung Lin $^{\mathrm{c}}$ \\ ${ }^{a}$ Department of Physics, National Taiwan Normal University, Taipei 116, Taiwan, ROC \\ ${ }^{\mathrm{b}}$ Department of Physics, National Kaohsiung Normal University, Kaohsiung 824, Taiwan, ROC \\ ${ }^{\mathrm{c}}$ Graduate Institute of Electronic Engineering and Department of Electrical Engineering, National Taiwan University, Taipei 116, Taiwan, ROC
}

Received 6 October 2005

\begin{abstract}
The modulated optical responses of InGaNAs/GaAs quantum well structures were characterized by photoreflectance spectroscopy. The quantum well excitonic interband transitions were observed in the spectral range above $h v=E_{\mathrm{g}}$ (InGaNAs). When the InGaNAs well is sandwiched in between GaNAs strain relief buffer layers, the quantum confinement potential becomes one step wider and increases the number of quantum states in the system. The quantum well subband energies and wave functions were calculated numerically to compare with the major optical features. The modulated optical responses are enhanced, especially for those quantum states with wave function extending into GaNAs and GaAs barriers where interface space charges induce strong internal field and enhance the electro-modulation efficiency.
\end{abstract}

(C) 2007 Elsevier Ltd. All rights reserved.

Keywords: A. Quantum well; A. Semiconductors; D. Optical properties

\section{Introduction}

The band gap of nitrogen containing III-V alloys like GaInNAs and GaNAs can be reduced by small amount of nitrogen content. These alloys have drawn considerable attention recently because of their interesting physical properties and a wide range of possible optoelectronic applications [1-3]. Many imaginative band gap and strain engineering designs are proposed since the demonstration of laser diodes based on InGaNAs quantum well (QW) structures. Because In and $\mathrm{N}$ not only reduce the band gap of GaAs but also have opposite effect on the lattice constant, considerable flexibility may be achieved. As a matter of fact, adding nitrogen into the compressively strained $\mathrm{InGaAs} / \mathrm{GaAs} \mathrm{QW}$ can reduce the strain, lower the band gap, and increase the conduction band offset. But the optical quality of the material may be deteriorated

\footnotetext{
*Corresponding author. Fax: + 886229326408.

E-mail address: lupond@phy03.phy.ntnu.edu.tw (C.-R. Lu).
}

significantly and the threshold current density will be increased with increasing $N$ mole fraction. For better performance of InGaNAs/GaAs QW lasers, the nitrogen composition of InGaNAs should be very small, and this will lead to strain increasing for the adequate wavelength $[4,5]$. Inserting strain compensating buffer layers to the InGaNAs/ GaAs QW structure can extend the emission wavelength and reduce the average strain of the system [6,7]. However, the effect of inserting strain compensating GaNAs layers on the optical properties of InGaNAs/GaAs QW is not well understood and requires further experimental studies.

In this paper, we studied the effect of GaNAs strain relief buffer layers on the optical properties of InGaNAs/GaNAs/ GaAs QW structures by photoreflectance (PR) spectroscopy. The excitonic interband transitions of the InGaNAs/ GaNAs/GaAs QW systems were observed in the spectral range above $h v=E_{\mathrm{g}}$ (InGaNAs). The confinement potential of the system with strain compensating GaNAs barriers is lowered and broadened, therefore more quantum states and larger optical transition rates were observed. 


\section{Experimental}

The MBE grown InGaNAs/GaAs QW structures consist of a central $\mathrm{In}_{0.3} \mathrm{Ga}_{0.7} \mathrm{~N}_{0.028} \mathrm{As}_{0.972}$ well of $6 \mathrm{~nm}$, two GaAs barriers of $120 \mathrm{~nm}$ and InGaP cladding layers. The aluminum-free InGaP cladding layers not only cover the same range of direct band gap energies as $\mathrm{AlGaAs}$ cladding layers, but also have higher catastrophic optical damage limit, resistance to dark-line defect propagation, lower surface recombination currents, and fabrication advantages such as lower growth temperature, negligible surface oxidation and ease of selecting etching [8,9]. The strain relief $\mathrm{GaN}_{0.035} \mathrm{As}_{0.965}$ buffer layers in between the InGaNAs well and GaAs barriers were $10 \mathrm{~nm}$ thick. Samples were annealed at $700{ }^{\circ} \mathrm{C}$ for $20 \mathrm{~min}$ under $\mathrm{N}_{2}$ purge. In the PR experiment, an argon ion laser as the excitation source provided pumping photons. They generate free electronhole pairs to neutralize the space charge in the system, and thus modulate the internal field strength. The probe light was provided by a quartz halogen lamp and the wavelength was selected by the quarter meter monochromator. Using the phase lock-in technique, the electromodulated optical responses of the excitonic transition between the QW confined hole states and electron states were amplified.

\section{Results and discussions}

The PR spectrum of the InGaNAs/GaAs QW and the spectrum of the system with $10 \mathrm{~nm}$ strain compensating GaNAs barriers at $300 \mathrm{~K}$ are compared in Fig. 1. Pumping photons induced modulation of the internal electric field produces two types of electro-modulated line shape. When the internal electric field is strong, the band edge transition will exhibit Franz-Keldysh oscillation (FKO) features above the band gap energy $[10,11]$. When the internal electric field is weak, the modulated optical responses will be the derivative like line shape $[12,13]$. The FKO features above the band gap energy of GaAs were comparatively strong, and were reduced for clear viewing of the derivative-like QW transitions in Fig. 1.

Due to the quantum confinement enhancement, the excitonic interband transitions of the InGaNAs/GaAs QW systems are clearly resolved. Vertical bars in Fig. 1 indicate where QW transitions are expected. Since the confinement potential of the system with strain compensating GaNAs barriers is wider and lower, more quantum states and thus more spectral features are observed, and they are also red shifted. When the photon energy is below and near the band edge of the GaNAs and GaAs barriers, near edge resonance features are observed in the spectra in Fig. 1.

A matrix transfer algorithm [14] was used to calculate the InGaNAs/GaNAs/GaAs QW subband energies numerically. The wave functions of the $j$ th layer may be described by

$\varphi_{j}(z)=A_{j} \mathrm{e}^{p_{j}(z)}+B_{j} \mathrm{e}^{-p_{j}(z)}$,

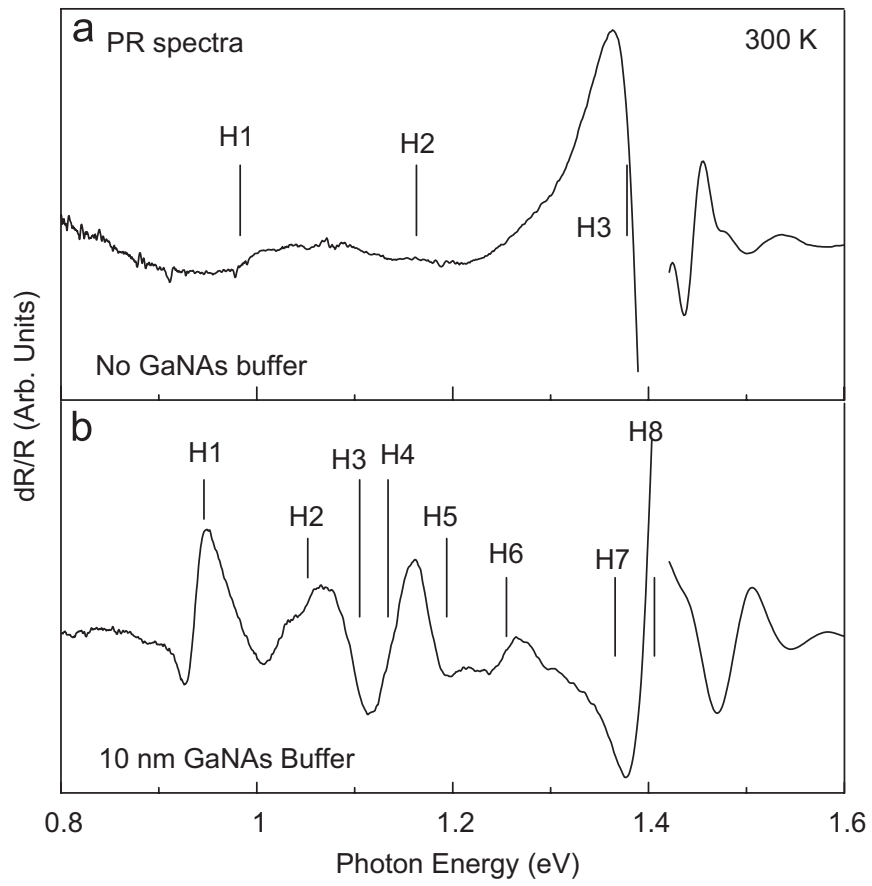

Fig. 1. Comparison of PR spectra at $300 \mathrm{~K}$ : (a) from the InGaNAs/GaAs QW and (b) from that with $10 \mathrm{~nm}$ GaNAs strain compensating buffer layers. FKO features above the band gap energy of GaAs are reduced for clear viewing of QW transitions. Vertical bars and H $n$ 's indicate expected transitions involving the $n$th heavy hole and electron subbands.

where $A_{j}$ and $B_{j}$ are probability amplitudes and $p_{j}$ are phase factors in the $j$ th layers. Express the boundary conditions in the matrix form, and then the amplitudes of the top and the bottom barriers are related by

$$
\left[\begin{array}{l}
A_{N} \\
B_{N}
\end{array}\right]=M_{N} M_{N-1} \cdots M_{1} M_{0}\left[\begin{array}{l}
A_{0} \\
B_{0}
\end{array}\right]=\left[\begin{array}{ll}
\alpha_{11} & \alpha_{12} \\
\alpha_{21} & \alpha_{22}
\end{array}\right]\left[\begin{array}{l}
A_{0} \\
B_{0}
\end{array}\right] .
$$

For bound states, their amplitudes should converge outside the QW region and both $A_{0}$ and $B_{N}$ should be zero. Varying the energy in the QW numerically, energies that make the matrix element $\alpha_{22}=0$ will be eigenenergies. The norm of $\alpha_{22}$ versus the electron energy of the InGaNAs/ GaAs QW with $10 \mathrm{~nm}$ GaNAs buffer layers is plotted in Fig. 2. When the energy equals one of the subband energies exactly, $\alpha_{22}$ would be zero. The subband energies correspond to the optical features indicated in Fig. 1(b).

Interband transitions involving the quantum-confined states of the InGaNAs/GaAs QW with and without strain compensating GaNAs barriers at $300 \mathrm{~K}$ are labeled in Fig. 1. The symbol $\mathrm{H} 1$ indicates the optical transitions involving the ground states of electron and heavy hole, and $\mathrm{H} 2$ indicates those involving the first excited states and so on. There are three electronic states confined in the InGaNAs/GaAs QW, and more quantum states in the system with $10 \mathrm{~nm}$ GaNAs buffer layers as observed in the spectra in Fig. 1(a) and (b), respectively. Theoretical studies have suggested that in the InGaNAs, the interaction between the nitrogen energy level and the conduction 


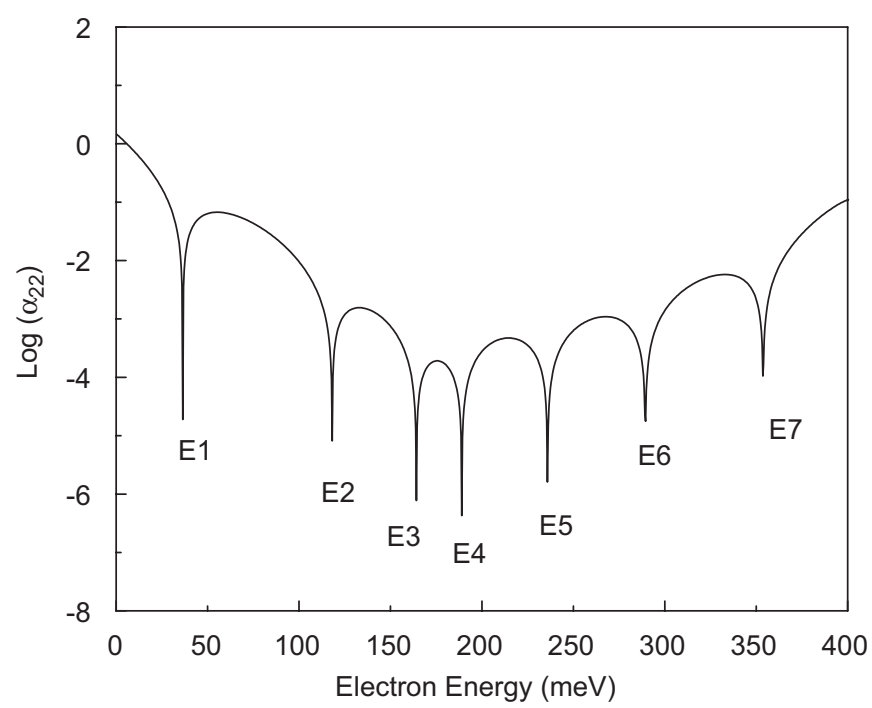

Fig. 2. The fourth matrix element $\alpha_{22}$ versus electron energy of the InGaNAs/GaAs QW with $10 \mathrm{~nm}$ GaNAs buffer layers. Each minimum should be zero at the exact electron eigenenergy.

band edge accounts for the compositional dependence of the electronic parameters [15-17]. The band gap energies and electron effective masses from the BAC model $[18,19]$ were the starting values in the calculation. For evaluating the InGaNAs/GaAs QW transition energies at $300 \mathrm{~K}$, the InGaNAs band gap energy was $0.86 \mathrm{eV}$ with a conduction band offset of $Q_{\mathrm{c}}=0.8$. The InGaNAs electron and heavy hole effective masses were $0.11 m_{\mathrm{e}}$ and $0.36 m_{\mathrm{e}}$, respectively. For those with GaNAs buffer layers, the band alignments were modified by the GaNAs band gap energy of $1.15 \mathrm{eV}$ with a small, about $10 \%$, valence band offset. The GaNAs electron and heavy hole effective masses were $0.12 m_{\mathrm{e}}$ and $0.45 m_{\mathrm{e}}$, respectively. The low energy transitions, $\mathrm{H} 1-\mathrm{H} 6$, in Fig. 1(b) are spectral signatures due to the inserting of GaNAs buffer layers, and depend weakly on the small perturbation of the GaNAs valence band due to nitrogen. Changing the sign or reducing the value of the GaNAs valence band offset influence the energy states near the GaAs band edge, and the effects are not clearly resolved because these weak transitions merge with the strong GaAs band edge transition.

Fig. 3 summarizes the electron probability amplitude distributions of the QW with no GaNAs buffer layers. The electron wave functions of the lowest two states are mostly confined in the narrow InGaNAs well region, and the optical responses are weak as shown in Fig. 1(a). The electron wave function of the third state has peaks near the InGaNAs/GaNAs interfaces and extends more into the GaAs region. The electro-modulation efficiency is strongly enhanced by the accumulation of space chargeinduced internal electric fields near the $\mathrm{GaAs} / \mathrm{InGaP}$ interface [20]. Therefore, the near-GaAs band edge transitions, $\mathrm{H} 3$ in Fig. 1(a) and $\mathrm{H} 8$ in Fig. 1(b), are comparatively strong. For the QW with $10 \mathrm{~nm}$ GaNAs buffer layers, electron wave functions of the lowest two

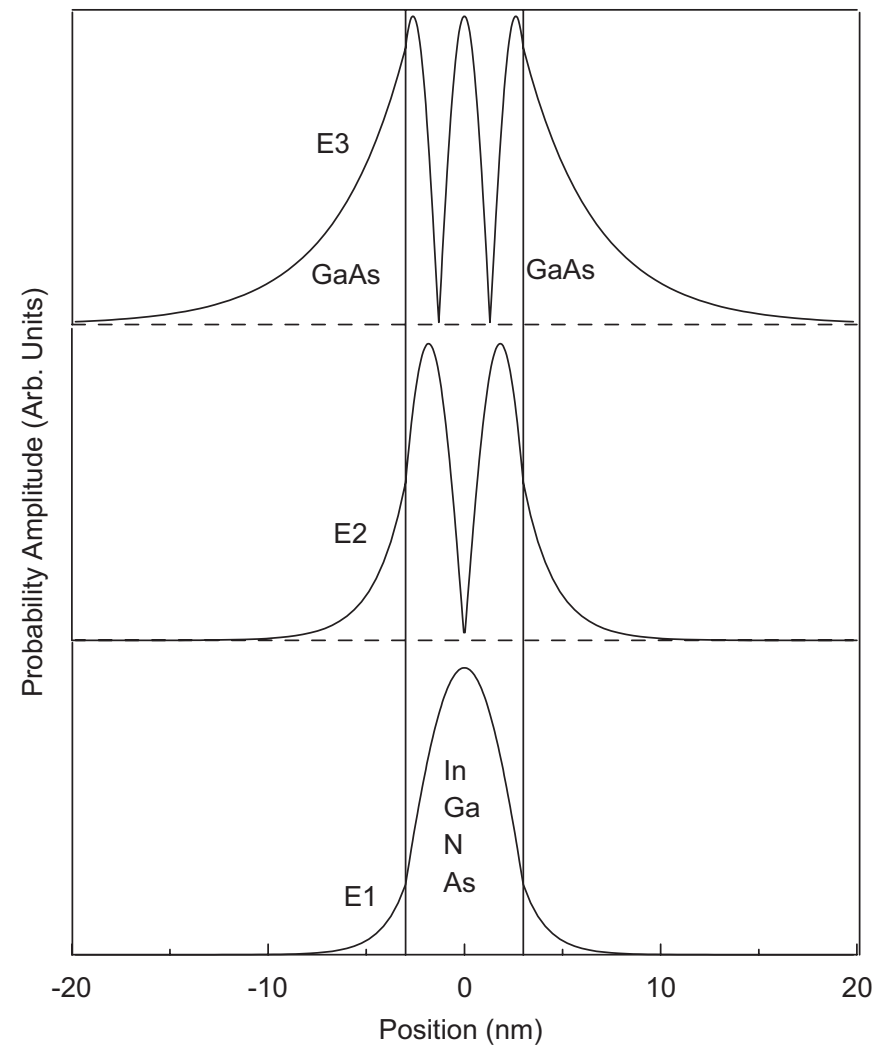

Fig. 3. The probability amplitudes of the electron states in InGaNAs/ GaAs QW. The electron wave function of the third electronic state has peaks near the InGaNAs/GaAs interface, penetrates further into the GaAs barriers and enhances the $\mathrm{H} 3$ transition in Fig. 1(a).

states are mostly confined in the narrow InGaNAs well region as shown in Fig. 4, and the modulated optical responses are weak as shown in Fig. 1(b). The electron wave functions of the third and the fourth states leak out of InGaNAs well and have a peak in the wider GaNAs buffer region as shown in Fig. 4. For these two states, the electromodulation efficiencies are enhanced by the space charges near the GaNAs/GaAs interfaces and correspond to the $\mathrm{H} 3$ and $\mathrm{H} 4$ transitions in Fig. 1(b).

\section{Conclusion}

We have investigated InGaNAs/GaAs and InGaNAs/ GaNAs/GaAs QW structures with InGaP cladding layers by PR spectroscopy. The excitonic interband transitions of the InGaNAs/GaNAs/GaAs QW systems were observed in the spectral range above $h v=E_{\mathrm{g}}$ (InGaNAs). The confinement potential of the system with strain compensating GaNAs barriers is one step wider; therefore, more quantum states and larger optical transition rates were observed. A matrix transfer algorithm was used to calculate the QW subband energies numerically. Band gap energies and effective masses were adopted from the band anti-crossing model and adjusted to obtain the subband energies to compare with the observed optical transitions. The PR spectral feature below and near the 


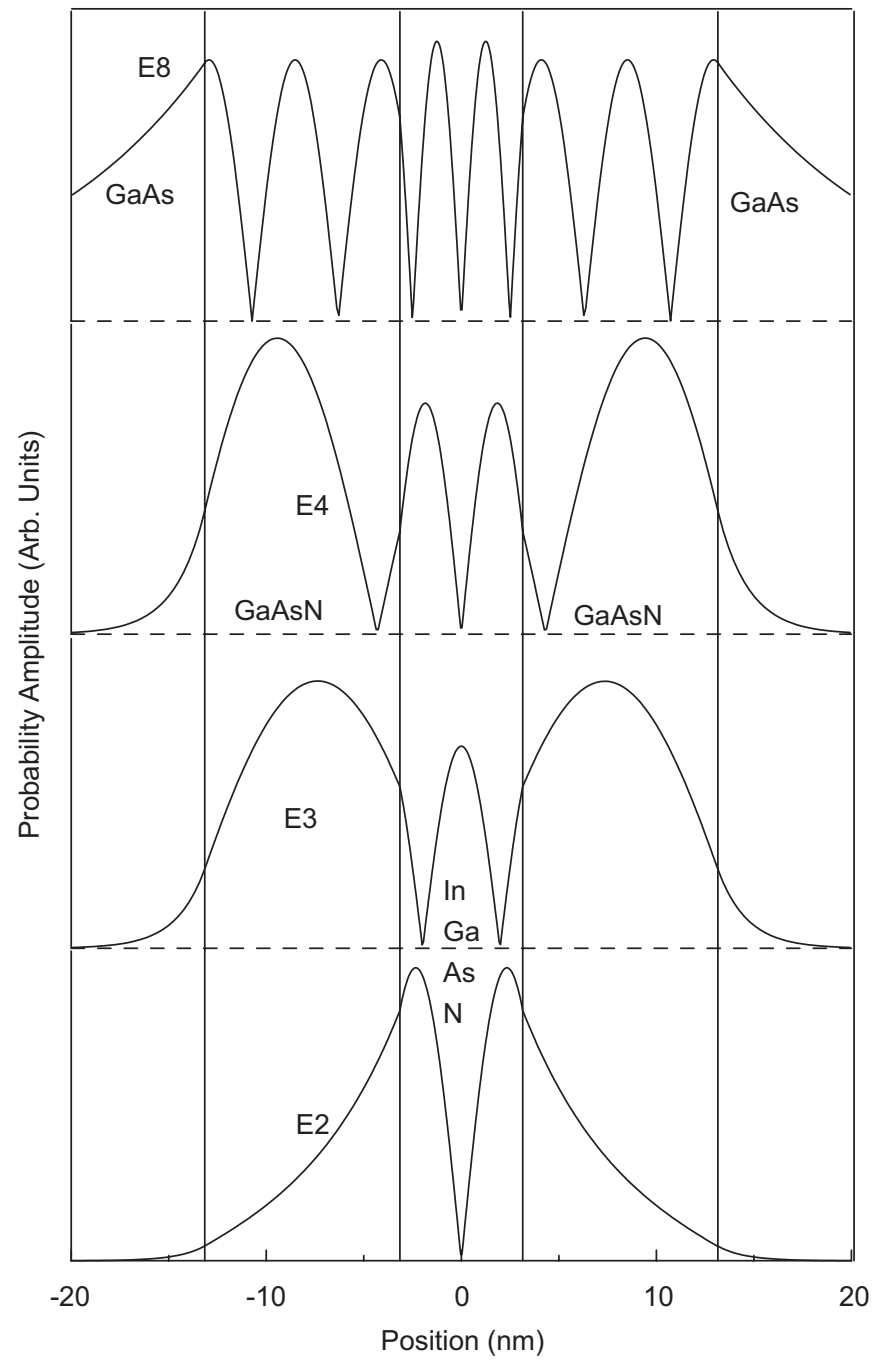

Fig. 4. The probability amplitudes of the electron states in InGaNAs/ GaAs QW with $10 \mathrm{~nm}$ GaNAs buffer. The electron wave functions of the third and the fourth states have peaks in the GaNAs buffer layers and enhance the $\mathrm{H} 3$ and $\mathrm{H} 4$ optical transitions in Fig. 1(b).

band edges of GaNAs buffer and GaAs barriers are enhanced by the electro-modulation due to the internal field near the GaNAs/GaAs and GaAs/InGaP interfaces.

\section{Acknowledgments}

Financial supports from National Science Council and Taiwan Normal University are gratefully acknowledged.

\section{References}

[1] M. Hetterich, M.D. Dawson, A.Yu. Egorov, D. Bernklau, H. Riechert, Electronic states and band alignment in GalnNAs/ GaAs quantum-well structures with low nitrogen content, Appl. Phys. Lett. 76 (2000) 1030.
[2] A. Lindsay, E.P. O'Reilly, Theory of enhanced bandgap nonparabolicity in $\mathrm{GaN}_{x} \mathrm{As}_{1-x}$ and related alloys, Solid State Commun. 112 (1999) 443.

[3] C. Ellmers, F. Hohnsdorf, J. Hoch, C. Agert, S. Leu, D. Karaiskaj, M. Hoffman, W. Stolz, W.W. Ruhle, Ultrafast (GaIn)(NAs)/GaAs vertical-cavity surface-emitting laser for the $1.3 \mu \mathrm{m}$ wavelength regime, Appl. Phys. Lett. 74 (1999) 2271.

[4] W. Li, J. Turpeinen, P. Melanen, P. Savolainen, P. Uusimaa, M. Pessa, Effects of rapid thermal annealing on strain-compensated GaInNAs/GaAsP quantum well structures and lasers, Appl. Phys. Lett. 78 (2001) 91.

[5] L.F. Bian, D.S. Jian, S.L. Lu, J.S. Huang, K. Chang, L.H. Li, J.C. Harmand, The effect of inserting strain-compensated GaNAs layers on the luminescence properties of GaInNAs/GaAs quantum well, J. Cryst. Growth 250 (2003) 339.

[6] W. Li, T. Jouhti, C.S. Peng, J. Konttinen, P. Laukkanen, E-M Pavelescu, M. Dumitrescu, M. Pessa, Low-threshold-current $1.32-\mu \mathrm{m}$ GaInNAs/GaAs single-quantum-well lasers grown by molecular-beam epitaxy, Appl. Phys. Lett. 79 (2001) 3386.

[7] E. Tournié, M.-A. Pinault, A. Guzmán, Mechanisms affecting the photoluminescence spectra of GaInNAs after post-growth annealing, Appl. Phys. Lett. 80 (2002) 4148.

[8] I. Eliashevich, J. Diaz, H. Yi, A. Wang, M. Razeghi, Reliability of aluminum-free $808 \mathrm{~nm}$ high-power laser diodes with uncoated mirrors, Appl. Phys. Lett. 66 (1995) 3087.

[9] N.T. Yeh, W.S. Liu, S.H. Chen, P.C. Chiu, J.I. Chyi, InAs/GaAs quantum dot lasers with InGaP cladding layer grown by solid-source molecular-beam epitaxy, Appl. Phys. Lett. 80 (2002) 535.

[10] D.E. Aspnes, A.A. Studna, Schottky-barrier electroreflectance: application to GaAs, Phys. Rev. B 7 (1973) 4605.

[11] R.N. Bhattacharys, H. Shen, P. Parayanthal, F.H. Pollak, T. Coutts, H. Aharoni, Electroreflectance and photoreflectance study of the space-charge region in semiconductors: (In-Sn-O)/InP as a model system, Phys. Rev. B 37 (1988) 4044.

[12] H. Shen, P. Parayanthal, F.H. Pollak, M. Tomkiewicz, T. Drommond, J.N. Schulman, Photoreflectance study of GaAs/AlAs superlattices: fit to electromodulation theory, Appl. Phys. Lett. 48 (1986) 653.

[13] B.V. Shanabrook, O.J. Glembocki, W.T. Beard, Photoreflectance modulation mechanisms in $\mathrm{GaAs}-\mathrm{Al}_{x} \mathrm{Ga}_{1-x} \mathrm{As}$ multiple quantum wells, Phys. Rev. B 35 (1987) 2540.

[14] M.O. Vassel, J. Lee, H.F. Lockwood, Multibarrier tunneling in $\mathrm{Ga}_{1-x} \mathrm{Al}_{x} / \mathrm{GaAs}$ heterostructures, J. Appl. Phys. 54 (1983) 5206.

[15] L. Bellaiche, S.H. Wei, A. Zunger, Composition dependence of interband transition intensities in GaPN, GaNAs, and GaPAs alloys, Phys. Rev. B 56 (1997) 10233.

[16] A. Lindsay, E.P. O'Reilly, Influence of nitrogen resonance states on the electronic structure, Solid State Commun. 118 (2001) 313.

[17] C. Skierbiszewski, P. Perlin, P. Wisniewski, W. Knap, T. Suski, W. Walukeiwicz, W. Shan, K.M. Yu, J.W. Ager, E.E. Haller, J.F. Geisz, J.M. Olson, Large, nitrogen-induced increase of the electron effective mass in $\mathrm{In}_{y} \mathrm{Ga}_{1-y} \mathrm{~N}_{x} \mathrm{As}_{1-x}$, Appl. Phys. Lett. 76 (2000) 2409.

[18] W. Shan, W. Walukeiwicz, J.W. Ager III, E.E. Haller, J.F. Geisz, D.J. Friedman, J.M. Olson, S.R. Kurtz, Band anticrossing in GaInNAs alloys, Phys. Rev. Lett. 82 (1999) 1221.

[19] W. Shan, W. Walukiewicz, K.M. Yu, J.W. Ager III, E.E. Haller, J.F. Geisz, D.J. Friedman, J.M. Olson, S.R. Kurtz, Effect of nitrogen on the electronic band structure of group III-N-V alloys, Phys. Rev. B $62(2000) 4211$.

[20] P. Krispin, A. Knauer, S. Gramlich, Electric field-induced redistribution of free carriers at isotype (In, Ga)P/GaAs interfaces, Mater. Sci. Eng., B 88 (2002) 129. 